\title{
PARAFORMALDEHYDE-GLUTARALDEHYDE AS A ROUTINE PHYTOPLANKTON FIXATIVE
}

\author{
Diane Lazinsky and Linda Sicko-Goad \\ Great Lakes Research Division, The University of Michigan, Ann Arbor, MI 48109, U.S.A.
}

Several fixatives such as Lugol's iodine solution and osmium tetroxide traditionally have been employed in phytoflagellate studies. Lugol's iodine (LI) stains cytoplasmic components, making the algae easier to study with a light microscope while at the same time it preserves their flagella. However, this fixative is not suitable for electron microscopy. Osmium tetroxide (Os) is also an excellent fixative for flagella (Rosowski, 1977). But due to its extreme toxicity, it is not useful for shipboard sampling and sample transport. Since we have successfully employed paraformaldehyde-glutaraldehyde (PFG) modified after Karnovsky (1965) in our laboratory for ultrastructural and cytochemical studies of cultured diatoms, we attempted to use this fixative for routine light and electron microscopic study of phytoflagellates to avoid toxicity problems and provide samples that would be useful for light microscopy, SEM, and TEM.

Unialgal cultures of Pandorina and Euglena were employed in the present study and fixed by one of the following methods:
(1) PFG $1 \%$ paraformaldehyde, $1 \%$ glutar- aldehyde, and $0.05 \mathrm{M}$ sodium caco- dylate

(2) GA $3 \%$ glutaraldehyde and $0.05 \mathrm{M}$ sodium cacodylate
(3) LI Lugol's iodine solution, $0.06 \%$

(4) Os $1 \%$ osmium tetroxide and $0.05 \mathrm{M}$ sodium cacodylate.

Routine examination of phytoplankton samples fixed with glutaraldehyde revealed that some flagella were intact on the phytoflagellates. However, neither the number of flagella, nor the organisms possessing the flagella responded in any predictable manner. Lugol's iodine was slightly better in that the cultured flagellates possessed flagella. However, these were not always intact, and higher concentrations of Lugol's removed the flagella completely. Of all fixatives employed, osmium tetroxide and PFG provided the most consistent results, with excellent flagellar preservation (Figs. 1 and 2). We have been utilizing PFG for fixing natural phytoplankton assemblages and find that the flagella of some of the more common genera such as Cryptomonas, Chroomonas, and Rhodomonas are intact, making the flagellate component of the assemblage easier to identify and study.

\section{REFERENCES}

Rosowski, J. R., 1977. J. Phycol., 13: 323.

Karnovsky, M. J., 1965. J. Cell Biol., 27: 137a 

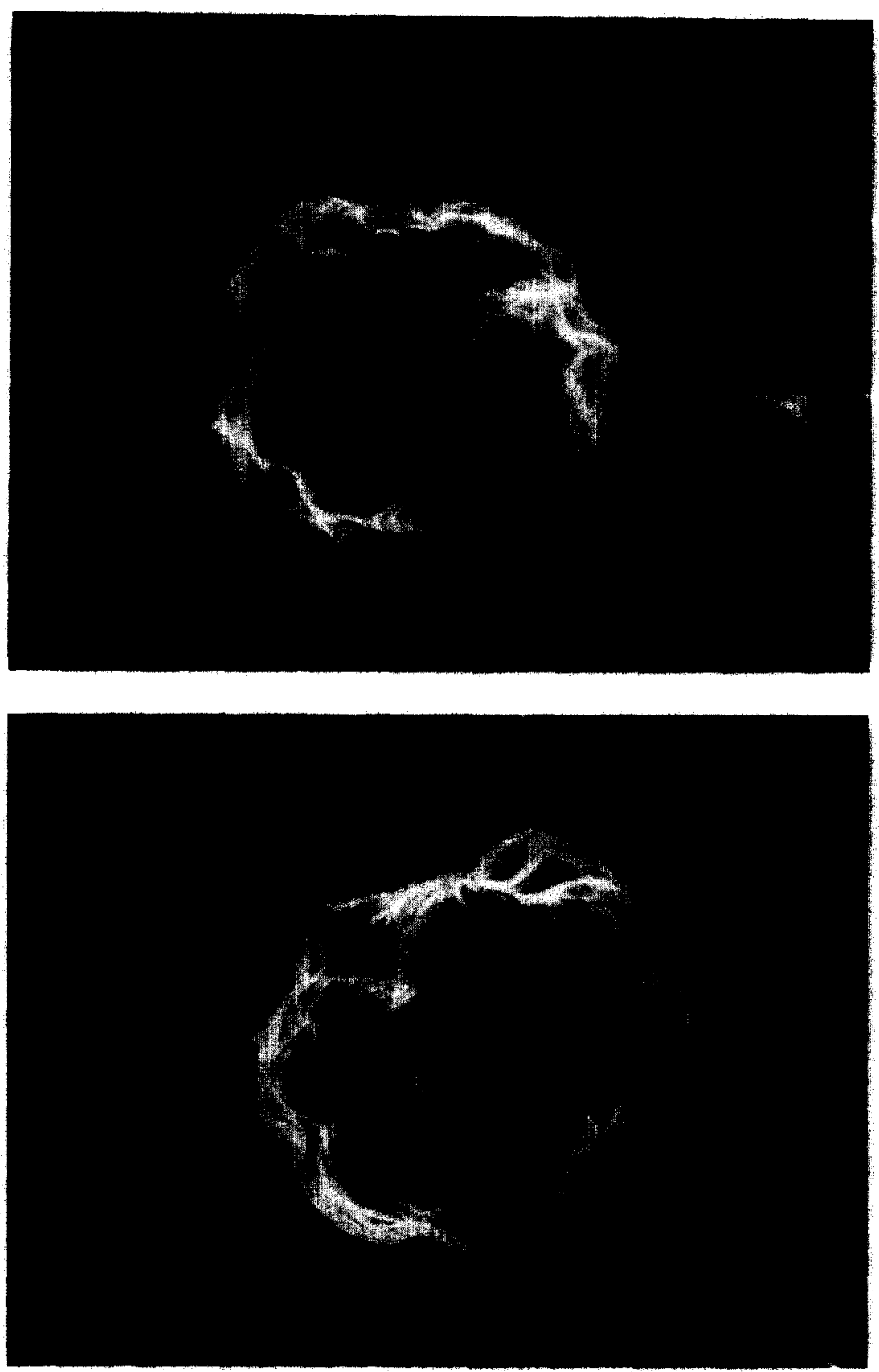

Fig. 1. SEM of Pandorina colony. Osmium fixation for $5 \mathrm{~min}$.

Fig. 2. SEM of Pandorina colony. PFG fixation for $\frac{1}{2} \mathrm{hr}$ at $4^{\circ} \mathrm{C}$. Both samples were dehydrated in 50, 95 and $100 \%$ alcohol, placed in Freon 113, and critical point dried in Freon 13. The samples were sputter coated with $20 \mathrm{~nm}$ gold. 